\title{
GALAXY STRATEGY FOR LIGO-VIRGO GRAVITATIONAL WAVE COUNTERPART SEARCHES
}

\author{
Neil Gehrels $^{1}$, John K. Cannizzo ${ }^{2,3}$, Jonah Kanner ${ }^{4}$, Mansi M. Kasliwal ${ }^{5}$, Samaya Nissanke ${ }^{6}$, and Leo P. Singer ${ }^{1,7}$ \\ ${ }^{1}$ NASA Goddard Space Flight Center, Mail Code 661, Greenbelt, MD 20771, USA \\ ${ }^{2}$ CRESST and Astroparticle Physics Laboratory, NASA/GSFC, Greenbelt, MD 20771, USA \\ ${ }^{3}$ Department of Physics, University of Maryland, Baltimore County, 1000 Hilltop Circle, Baltimore, MD 21250, USA \\ ${ }^{4}$ LIGO, California Institute of Technology, Pasadena, CA 91125, USA \\ ${ }^{5}$ Observatories of the Carnegie Institution for Science, 813 Santa Barbara Street, Pasadena, CA 91101, USA \\ ${ }^{6}$ Institute of Mathematics, Astrophysics and Particle Physics, Radboud University, Heyendaalseweg 135, 6525 AJ Nijmegen, The Netherlands \\ Received 2015 August 14; accepted 2016 February 5; published 2016 March 30
}

\begin{abstract}
In this work we continue a line of inquiry begun in Kanner et al. which detailed a strategy for utilizing telescopes with narrow fields of view, such as the Swift X-ray Telescope (XRT), to localize gravitational wave (GW) triggers from LIGO/Virgo. If one considers the brightest galaxies that produce $\sim 50 \%$ of the light, then the number of galaxies inside typical GW error boxes will be several tens. We have found that this result applies both in the early years of Advanced LIGO when the range was small and the error boxes were large, and will apply in the later years when the error boxes will be small and the range will be large. This strategy has the beneficial property of reducing the number of telescope pointings by a factor of 10-100 compared with tiling the entire error box. Additional galaxy count reduction will come from a GW rapid distance estimate which will restrict the radial slice in search volume. Combining the bright galaxy strategy with a convolution based on anticipated GW localizations, we find that the searches can be restricted to about $18 \pm 5$ galaxies for 2015 , about $23 \pm 4$ for 2017 , and about $11 \pm 2$ for 2020. This assumes a distance localization at the putative neutron star-neutron star merger range $\mu$ for each target year, and these totals are integrated out to the range. Integrating out to the horizon would roughly double the totals. For localizations with $r \ll \mu$ the totals would decrease. The galaxy strategy we present in this work will enable numerous sensitive optical and XRTs with small fields of view to participate meaningfully in searches wherein the prospects for rapidly fading afterglow place a premium on a fast response time.
\end{abstract}

Key words: galaxies: statistics - gamma-ray burst: general - gravitational waves - X-rays: general

\section{INTRODUCTION}

The advent of Advanced LIGO (aLIGO-Aasi et al. 2015) and Advanced Virgo (AdV-Acernese et al. 2015) heralds the dawn of a new age of discovery in which gravitational wave $(\mathrm{GW})$ detections will supplement traditional electromagnetic (EM) detections, such as those by Swift (Gehrels et al. 2004). We refer to aLIGO/AdV in combination as LVC. These GW observatories will begin operating soon at a fraction of design capability, and within a few years should be able to detect neutron star-neutron star (NS-NS) mergers out to $\sim 450 \mathrm{Mpc}$ and black hole-neutron star (BH-NS) mergers out to $\sim 900 \mathrm{Mpc}$ (Aasi et al. 2013). These distances are referred to as the "horizon" for these events. Although black hole-black hole (BH-BH) mergers are detectable by aLIGO/AdV to even greater distances, since we are focused in this work on GW signals with accompanying EM counterparts, they will not be a part of our discussion.

It is important to distinguish between "horizon" and "range." The "inspiral range," the most commonly cited figure-of-merit regarding LVC sensitivity, is defined as the radius of a sphere whose volume equals the sensitivity volume within which a signal-to-noise ratio $(\mathrm{S} / \mathrm{N}) \geqslant 8$ detection is achieved for a 1.4-1.4 $M_{\odot}$ NS-NS merger, averaged over all sky locations and binary inclinations. In this work we use $\mu$ to denote the NS-NS inspiral range. The antenna projection functions and associated averagings were discussed by Finn \& Chernoff (1993) and Finn (1996). The inspiral range is not a hard upper limit, as face-on binary orbit mergers produce stronger signals (Dalal

\footnotetext{
${ }^{7}$ NASA Postdoctoral Program.
}

et al. 2006; Maggiore 2007; Schutz 2011; Nissanke et al. 2013). The maximum theoretical detection distance, the horizon, is 2.26 times the range (Finn \& Chernoff 1993; see also Abadie et al. 2010). A simple calculation shows that in a general population with binaries of random inclinations and positions over the whole sky, the fraction of aLIGO detections one expects to pick up from beyond the nominal inspiral range is about half of the total (Nissanke et al. 2010, 2013; Singer 2015).

High $\mathrm{S} / \mathrm{N}$ detections will permit important physical parameters to be measured that are not easily accessible through traditional means, such as the masses and spins of the merger components and the luminosity distance $D_{L}$ to the merger. A joint $\mathrm{EM} / \mathrm{GW}$ detection would provide powerful constraints on the merger physics. Various groups have begun to the examine the prospects of finding an EM counterpart to a GW trigger (Nuttall \& Sutton 2010; Metzger \& Berger 2012; Nissanke et al. 2013; Hanna et al. 2014; Chen \& Holz 2015; Chan et al. 2015; Fan et al. 2015; Ghosh \& Nelemans 2015). Evans et al. (2016) consider strategies for X-ray observations using Swift/X-ray Telescope (XRT).

In Section 2 we present an overview of short GRBs, including putative GW rates for NS-NS mergers, and a discussion of "kilonova" emission accompanying the decay of radionuclides. In Section 3 we describe a galaxy catalog-the Census of the Local Universe (CLU) - and quantify its completeness out to distances relevant for aLIGO. In Section 4 we estimate galaxy totals that are concomitant with putative aLIGO error volumes for the next $5 \mathrm{yr}$, if one restricts attention to bright galaxies. In Section 5 we discuss potential EM observations in the optical, X-ray, and radio which might 
follow GW detection, and in Section 6 we summarize our findings.

\section{ALIGO-ADV (LVC) OBSERVATIONS}

\subsection{GW Radiation}

Astrophysical sources that are powerful in EM detectors are always weak in GW if the underlying physical process is nearly spherically symmetric. Supernovae (SNe) are among the brightest EM transients but might produce peak GW strain amplitudes of $|h| D \lesssim 10 \mathrm{~cm}$, where $h$ is the dimensionless strain and $D$ the distance to the source, and so may be observable in aLIGO only to a few kpc (Ott et al. 2013; see their Figure 14).

In fact the lowest order contributions to $\mathrm{GW}$ radiation arise from a changing mass quadrupole moment. As such, binary mergers of compact objects give by far the strongest signals, yielding peak GW strain amplitudes $|h| D \sim 1 \mathrm{~km}$. Belczynski et al. (2002) utilize a population synthesis code to delineate a range of rates for different types of binary mergers. They calibrate NS-NS merger rates using known galactic binary pulsars. For aLIGO they infer $1-400 \mathrm{yr}^{-1}$ for NS-NS, 9-400 $\mathrm{yr}^{-1}$ for BH-NS, and $0-8000 \mathrm{yr}^{-1}$ for BH-BH. Abadie et al. (2010) incorporate the results of the Belczynski et al. study and estimate an LVC NS-NS merger rate $\sim 40 \mathrm{yr}^{-1}$, with a range $\sim 0.4-400 \mathrm{yr}^{-1}$. Aasi et al. (2013) update the results of Abadie et al. (2010); they find a similar range in the rate for NS-NS mergers, but an average rate a factor of $\sim 2$ lower. A conservative lower limit to the NS-NS rate for a putative aLIGO inspiral range of $200 \mathrm{Mpc}$ is $\sim 3 \mathrm{yr}^{-1}$ (Phinney 1991; for a more up-to-date study, see O'Shaughnessy \& Kim 2010). More recently Dominik et al. (2013) calculate local (i.e., $z \simeq 0$ ) rates of $\sim 10^{2} \mathrm{Gpc}^{-3} \mathrm{yr}^{-1}$ for NS-NS mergers and $\sim 10 \mathrm{Gpc}^{-3} \mathrm{yr}^{-1}$ for BH-NS mergers. Given a putative LVC sensitivity volume $\sim 0.03 \mathrm{Gpc}^{3}$ relevant for a $200 \mathrm{Mpc}$ range, this translates into LVC detection rates of $\sim 3 \mathrm{yr}^{-1}$ and $\sim 0.3 \mathrm{yr}^{-1}$, respectively, which is similar to Phinney (1991). As indicated earlier, taking into account the more realistic totals achieved in mapping from range to horizon would roughly double these estimates. In this work we restrict our attention to NS-NS mergers.

\subsection{GRBs and Beamed EM Radiation}

GRBs come in two flavors: long $(>2 \mathrm{~s})$ and short $(<2 \mathrm{~s})$ (Kouveliotou et al. 1993). Long GRBs (IGRBs) are thought to arise from the explosion of a massive star-nearby lGRBs have associated SNe. More interesting for aLIGO are short GRBs (sGRBs), which are thought to be due to mergers involving at least one NS, i.e., either NS-NS or BH-NS mergers, for which we are placed along the binary rotation axis (Eichler et al. 1989; Narayan et al. 1992). The fact that lGRBs are mostly spherically or axially symmetric makes them less interesting than sGRBs vis a vis GW detections.

The observed redshift range is from about 0.2 to 2 for sGRBs, with a mean of about 0.4. The XRT commonly observes sGRBs up to $z \simeq 0.5$ but in $10.5 \mathrm{yr}$ of operation Swift has not seen one with a measured redshift of $z<0.1$, i.e., the NS-NS merger aLIGO target GW horizon $\sim 400 \mathrm{Mpc}$, suggesting that such events may be quite rare.

Estimates for jet beaming are $\theta_{\mathrm{j}} \sim 5^{\circ}$ for $1 \mathrm{GRBs}$ and $\theta_{\mathrm{j}} \sim 5^{\circ}-15^{\circ}$ for sGRBs (Burrows et al. 2006; Grupe et al. 2006; Fong et al. 2012). Beaming angles for sGRBs are still highly uncertain. The beaming factors $f_{b}=1-\cos \theta_{\mathrm{j}} \simeq \theta_{\mathrm{j}}^{2} / 2$ are roughly $1 / 300$ for 1GRBs and $1 /$ 30 for sGRBs. Based on the observed rate of sGRBs by Swift, Coward et al. (2012) estimate a LVC detection rate of $\sim 3-30 \mathrm{yr}^{-1}$ for $\theta_{\mathrm{j}} \simeq 15^{\circ}$. Chen \& Holz (2013) claim 3-7 $\mathrm{yr}^{-1}$ for GRB GW+EM detections. Kelley et al. (2013) estimate the rate of Swift or Fermi observations joined with LVC detections to be $\sim 0.07 \mathrm{yr}^{-1}$. Siellez et al. (2014) consider current and future high energy missions and estimate a rate of simultaneous GW+EM detections of $\sim 0.1-4 \mathrm{yr}^{-1}$ in the LVC era. Wanderman \& Piran (2015) estimate a co-detection rate $\mathrm{LVC}+$ Fermi of $0.1-1 \mathrm{yr}^{-1}$ and LVC + Swift of $0.02-0.14 \mathrm{yr}^{-1}$.

\subsection{Kilonova Emission}

Various groups have explored the $\mathrm{SN}$-like transient powered by radioactive decay of the spray of material $\sim 10^{-6}-10^{-1} M_{\odot}$ ejected from the NS (Eichler et al. 1989; Li \& Paczyński 1998; Metzger et al. 2010; Metzger \& Berger 2012). The resultant "kilonova" (dimmer than a SN and brighter than a nova) would produce relatively isotropic optical/NIR emission after a NSNS/BH-NS merger. While SN Ia light curves are powered primarily by decay of ${ }^{56} \mathrm{Ni}$, the ejecta from a disrupted NS is neutron rich and yields little Ni. Much heavier radioactive elements form via rapid neutron capture ( $r$-process) nucleosynthesis following the decompression of the ejecta from nuclear densities. These newly synthesized elements subsequently undergo nuclear fission $-\alpha$ and $\beta$ decays on much longer timescales. The resulting energy release can power detectable thermal emission once the ejecta expand sufficiently that photons can escape. Recent general relativistic NS-NS merger simulations (Bauswein et al. 2013) indicate that a small fraction of the ejecta, $\sim 10^{-4} M_{\odot}$, or a few percent, expand rapidly enough for most neutrons to escape capture. The $\beta$ decay of these free neutrons in the outermost ejecta powers a precursor to the main kilonova emission peaking on a timescale of hours after NS-NS merger (Metzger et al. 2015). For $D \simeq 200 \mathrm{Mpc}$ this emission peaks in the $U$-band $(\sim 365 \mathrm{~nm})$ at $m_{U} \simeq 22$ (Metzger et al. 2015).

Roberts et al. (2011) carry out self-consistent calculations from merger through to full three-dimensional (3D) radiation transport using simplified opacities. They highlight asymmetries in the NS-NS merger. Kasen et al. (2013) argue that the opacity of the expanding $r$-process material is dominated by bound-bound transitions from those ions with the most complex valence electron structure, i.e., the lanthanides (Tanaka \& Hotokezaka 2013; Grossman et al. 2014; Wanajo et al. 2014). They compute atomic structure models for a few representative ions in order to calculate the radiative transition rates for tens of millions of lines, and find that resulting $r$ process opacities are orders of magnitude larger than those of ordinary (e.g., iron-rich) SN ejecta. The resultant light curves should be longer, dimmer, and redder than previously thought. The spectra have broad absorption features and peak in the IR $(\sim 1 \mu \mathrm{m})$. Kasen et al. (2015) combine two-dimensional hydrodynamical disk models with wavelength-dependent radiative transfer calculations to generate model light curves and spectra. They discern two components to the kilonova light curve, a blue optical transient ( 2d) arising from the outer lanthanide-free ejecta and an IR transient $(\sim 10 \mathrm{~d})$ coming from the inner, lanthanide line-blanketed region. There had been an earlier suggestion of these two components in work by Barnes $\&$ Kasen (2013) using a less sophisticated model. 
Specific predictions for kilonova light curves are dependent on uncertainties such as the type of ejecta (dynamical and/or disk outflows), ejecta masses, and velocities. Recent time dependent calculations find ejecta masses in the range $\sim 10^{-3}-10^{-1} M_{\odot}$ and velocities $\sim 0.1-0.3 c$ (e.g., Foucart et al. 2011, 2015; East \& Pretorius 2012; Fernández \& Metzger 2013; Hotokezaka et al. 2013; Kyutoku et al. 2013; Piran et al. 2013; Fernández et al. 2015; Sekiguchi et al. 2015). Assuming iron-rich SN ejecta, Metzger \& Berger (2012) predict peak optical luminosities of $\sim 10^{41}-10^{42} \mathrm{erg} \mathrm{s}^{-1}$ and concomitant $M_{R}$ values of -14 to -17 . For $M_{\text {eject }}=10^{-2} M_{\odot}$ and $v_{\text {ejecta }}=0.1 c$, Barnes \& Kasen (2013) calculate a peak absolute magnitude in the near-IR $(\lambda \simeq 1.7 \mu \mathrm{m})$ of $M_{H}=-15.5$, in good agreement with Tanaka \& Hotokezaka (2013), Tanaka et al. (2014), and Grossman et al. (2014).

GRB 130603B might be the first detected kilonova (Berger et al. 2013; Tanvir et al. 2013). It was a short GRB at $z=0.356$ with a duration $\sim 0.2 \mathrm{~s}$ in the BAT. If correct, it would confirm that compact-object mergers are the progenitors of short GRBs and also the sites of significant production of $r$-process elements.

In addition to the optical/near-IR kilonova emission, one also expects a characteristic signal in the radio (Nakar \& Piran 2011; Hotokezaka \& Piran 2015) as the ejecta first interact internally and then externally with the ISM. The latter interaction gives rise to a blast wave with concomitant enhancement of magnetic fields and electron acceleration, leading to synchrotron radiation and radio emission. Three temporal components to the radio band have been considered and studied: (i) early-time anisotropic emission along the relativistic jet axis associated with the ultrarelativistic ejecta, (ii) mildly relativistic, quasi-isotropic emission accompanying cocoon-breakout, leading to potential radio flares for off-axis observers, and (iii) late-time sub-relativistic dynamical ejecta producing radio flares on timescales of years. The latter emission should be nearly isotropic, and provide standard calorimetry on the global energetics of the initial explosive event, just as has been the case for long GRBs.

\section{CLU CATALOG}

Any given galaxy catalog is generally not optimal for GW follow-up studies. Consider two extremes: The Two Micron All Sky Survey (2MASS) survey (Skrutskie et al. 2006) has good coverage in both the northern and southern skies, but does not go very deep (Huchra et al. 2012). The Millenium Galaxy Catalog, comprising spectroscopic redshifts of galaxies from $2 \mathrm{dF}$ or SDSS, is deep, but covers only a small slice along the celestial equator (Driver et al. 2005). An attempt to overcome these limitations led to the Gravitational Wave Galaxy Catalog $=$ GWGC (White et al. 2011). Its only limitation for our current study is that it does not extend beyond $100 \mathrm{Mpc}$. One of us (M. M. Kasliwal et al. 2016, in preparation) has amassed a catalog based on the union of several existing catalogs - the CLU—which is suitable for GW +EM follow-up studies. As we shall show, for bright galaxies the CLU is complete out to the anticipated aLIGO GW inspiral range for NS-NS mergers up to 2020.

In order to show completeness, we must adopt a model for galaxy number density in the local universe. The Schechter luminosity function (Schechter 1976) provides a useful description of the space density of galaxies as a function of their luminosity, $\rho_{\text {gal }}(x) d x=\phi^{*} x^{a} e^{-x} d x$, where $x=L / L^{*}$ and $L^{*}$ is a characteristic galaxy luminosity where the power-law form of the function truncates. It has proven to be applicable over up to 10 mag in deep surveys (e.g., Bonne et al. 2015). The CLU catalog is amassed from many different surveys. One

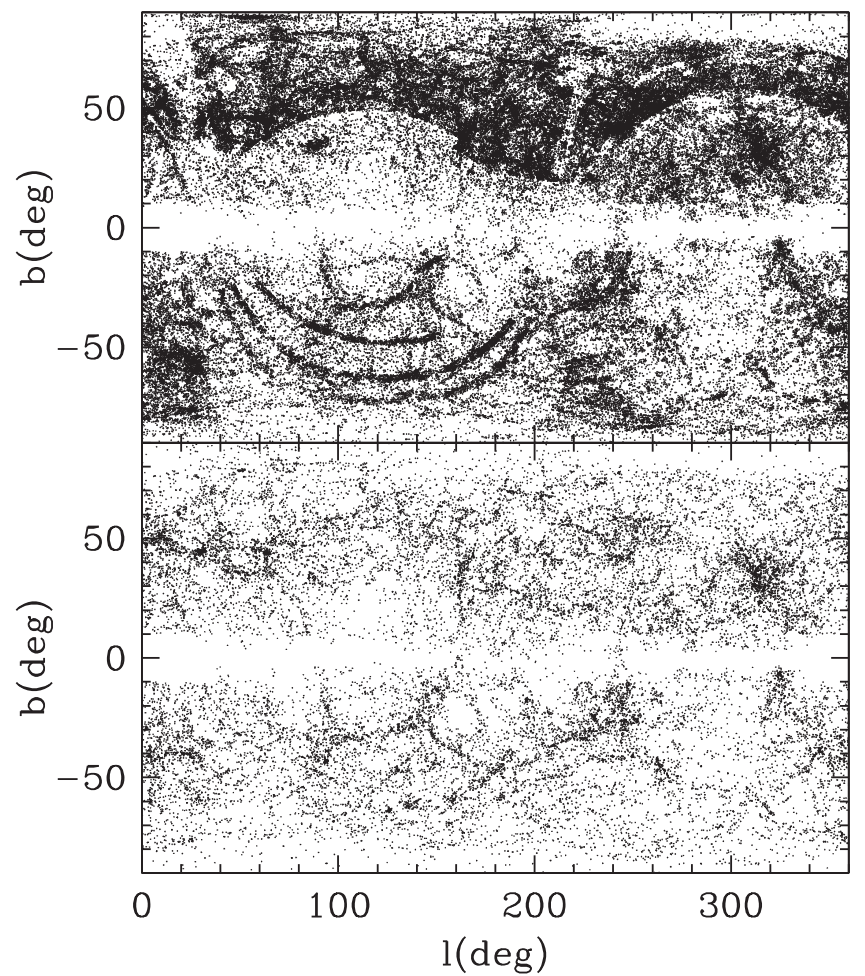

Figure 1. Sky maps of the CLU catalog in galactic coordinates. Shown are all 144214 galaxies (upper panel), and only the 27559 galaxies for which $L>L_{B}^{*}$ (lower panel). The swath of incompleteness in both panels represents the galactic plane, which is excluded in many surveys. Since its total area is small, we do not include any exclusion factor in solid angle for this region.

of the CLU data columns, $b_{\mathrm{tc}}$, consists of apparent $B$ magnitudes $m_{B}$ for entries where they are available, and pseudo- $m_{B}$ values for sources from other bands, for instance 2MASS. Hence for this work $\left(\phi^{*}, L, L^{*}\right) \rightarrow\left(\phi_{B}{ }^{*}, L_{B}, L_{B}{ }^{*}\right)$, Physically, $L_{B}^{*}$ represents the turn-over in the distribution between a power-law for low $x$ and an exponential for high $x$.

We adopt the following values derived from $B$-band measurements of nearby field galaxies: $\phi^{*}=(1.6 \pm 0.3) \times$ $10^{-2} h^{3} \mathrm{Mpc}^{-3}, \quad a=-1.07 \pm 0.07, \quad L_{B}^{*}=(1.2 \pm 0.1) \times$ $10^{10} h^{-2} L_{B, \odot}$, with a corresponding $M_{B}^{*}=-19.7 \pm 0.1+$ $5 \log _{10} h=-20.47 \quad$ (e.g., Norberg et al. 2002; Liske et al. 2003; González et al. 2006, and references therein). By comparison, for the Milky Way galaxy $M_{B}=-20.42$. This is based on a Milky Way $B$-band luminosity $2.3 \times 10^{10} L_{B, \odot}$ (Carroll \& Ostlie 1996), where $L_{B, \odot}$ is the solar $B$-band luminosity, and an absolute solar $B$ magnitude of 5.48 (Allen 1973). We take $h=0.7$ based on the latest weighted overlap between the Planck results and the rest of astronomy (Ade et al. 2014).

Integrating over luminosity gives integrated number density $\rho_{0, \text { gal }}=\int_{x_{1}}^{\infty} \rho_{\text {gal }}(x) d x$. Although $\rho_{0, \text { gal }} \rightarrow \infty$ as $x_{1} \rightarrow 0$ for $a<-1$, the integrated luminosity density diverges only for $a<-2$. One has

$$
\int_{x_{1}}^{\infty} \phi^{*} L^{*} x^{a+1} \exp (-x) d x=\phi^{*} L^{*} \Gamma\left(a+2, x_{1}\right)
$$

where $\Gamma$ is the incomplete gamma function. For $a=-1$ the total luminosity density is $\phi^{*} L^{*} \Gamma(2+a)=\phi^{*} L^{*}=1.9 \times$ $10^{8} h L_{B, \odot} \mathrm{Mpc}^{-3}$. Dividing by a Milky Way $B$-band luminosity yields a density $\sim 6 \times 10^{-3} \quad \mathrm{MWE} \mathrm{Mpc}^{-3}$, where 


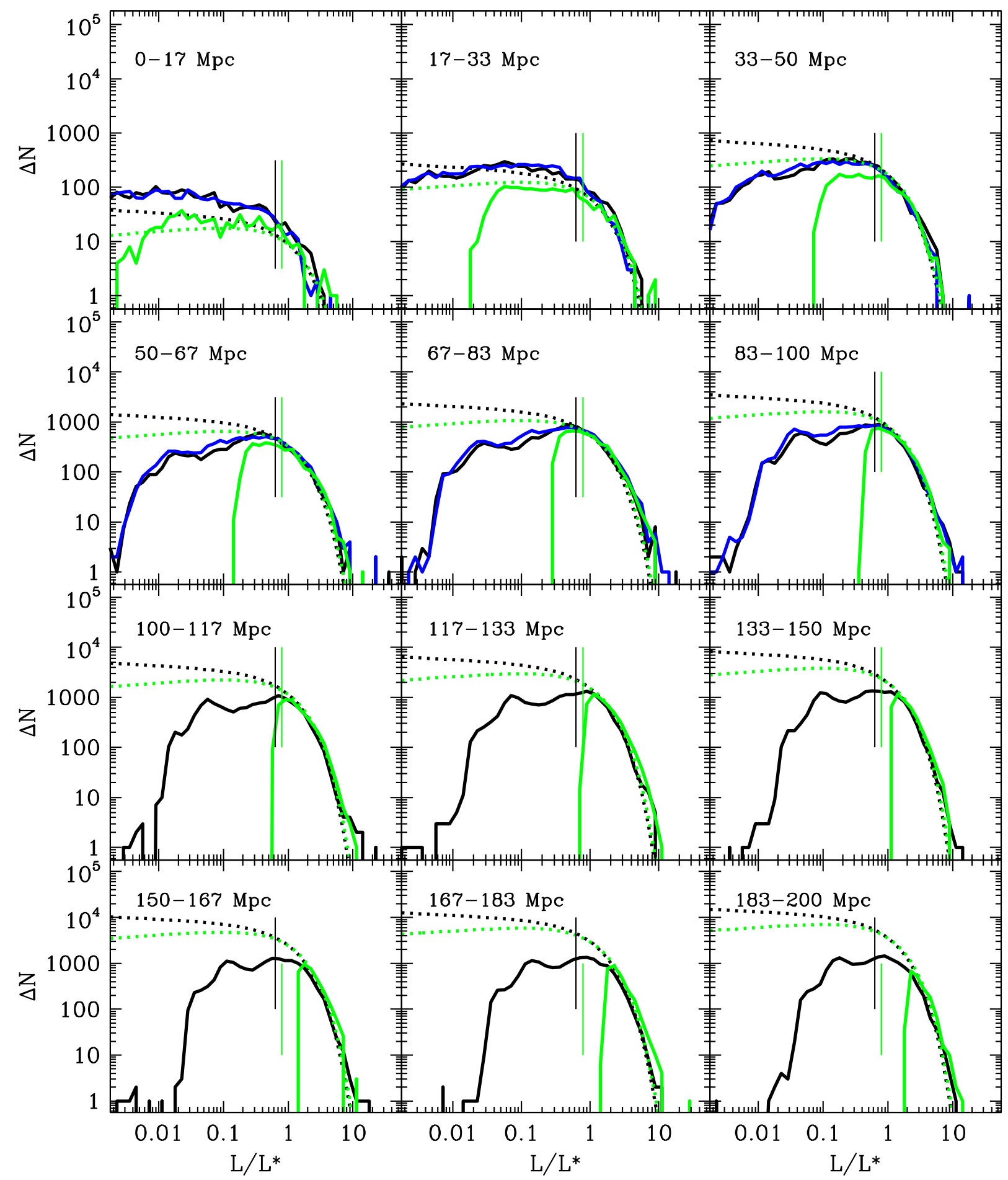

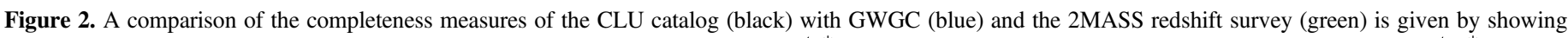

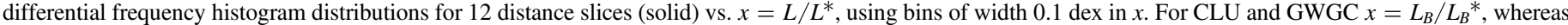

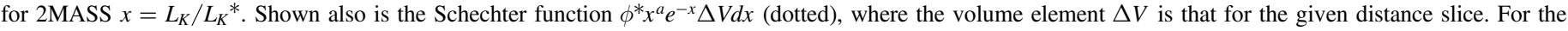
2MASS data, which are in the $K$-band, $\alpha=-0.9$ and $x_{1 / 2}=0.790$. The vertical line segments in each panel indicate $x=x_{1 / 2}$.

MWE $=$ Milky Way equivalent galaxy. For $a=-1$, half of the luminosity density is contributed by galaxies with $x_{1 / 2}>$ 0.693 . For the power law of interest in this study, $a=-1.07$, the cutoff lies at $x_{1 / 2}>0.626$, or $M_{B} 1 / 2=-19.97$. This corresponds to $\sim 0.66$ of the Milky Way luminosity. To arrive at this $x_{1 / 2}$ value we used the fact that $\int_{x_{1}}^{\infty} x^{a+1} e^{-x} d x=$
1.04559 for $x_{1}=0$ and $a=-1.07$, and half this value $1.04559 / 2$ is achieved for $x_{1}=0.626$.

Figure 1 presents sky maps of the CLU catalog, showing all the galaxies, and also those for which $x>1$, where $x=L_{B} / L_{B}{ }^{*}$. The dark strips evident in the top panel indicate individual deep surveys which make up the CLU. Restricting the sample to 


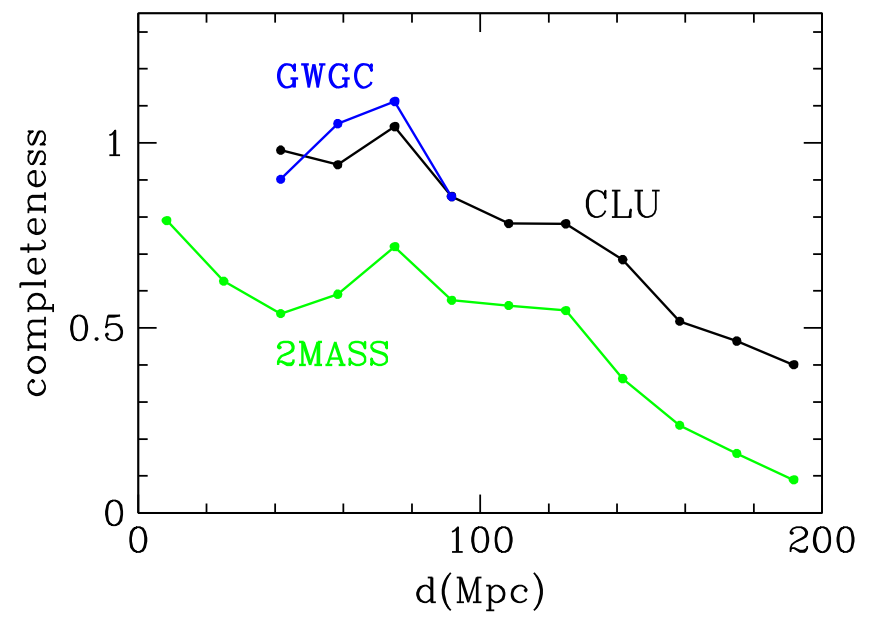

Figure 3. Completeness relative to the Schechter function for $x>x_{1 / 2}$ for CLU (black), GWGC (blue), and 2MASS (green) using the data presented in Figure 2.

intrinsically bright galaxies cleans it up considerably and reveals large scale structure.

Figure 2 provides a measure of the completeness as gauged by the Schechter function. We compare the CLU catalog with the GWGC and 2MASS redshift survey out to $200 \mathrm{Mpc}$ using 12 radial slices. Within each slice we bin the data in $x=L / L^{*}$ and compare with the Schechter function weighted by the volume of the given slice. For CLU and GWGC $x=L_{B} / L_{B}{ }^{*}$, whereas for 2MASS $x=L_{K} / L_{K}{ }^{*}$. We adopt $M_{K}{ }^{*}=$ $-23.1+5 \log _{10} h=-23.87$. Since GWGC ends at $100 \mathrm{Mpc}$ there are no data in the more distant bins. For all three catalogs there is a progressive loss of fainter galaxies with distance relative to Schechter. For all radii the CLU follows the Schechter function for $x \gtrsim x_{1 / 2}$. In some of the panels in Figure 2 one sees spikes at $x \simeq 10$. These may be due to misidentified local objects spuriously placed at larger distances.

In this paper we propose to utilize the brighter galaxies in each bin, which tends to mitigate the effects of incompleteness brought about by a progressive loss of fainter galaxies with increasing distance. The CLU catalog is fairly complete relative to Schechter above $x_{1 / 2}$. This is shown in Figure 3, where we compare with GWGC and 2MASS. In this work we will argue for including only the brighter galaxies in GW+EM follow-up studies. As noted earlier, this implies cutting galaxies below $x_{1 / 2}$. For the three fiducial distances of interest in the next section, $60 \mathrm{Mpc}, 120 \mathrm{Mpc}$, and $180 \mathrm{Mpc}$, the CLU catalog is complete above this cut line at a level of $\sim 100 \%, \sim 80 \%$, and $\sim 40 \%$, respectively. We stress that this completeness only includes galaxies for which $x>x_{1 / 2}$. Since these three distances are based on the NS-NS inspiral ranges for 2015, 2017, and 2020, an additional factor which must be included for the third year stems from the fact that the CLU catalog ends at $200 \mathrm{Mpc}$, namely the fact that about half the detections would be expected to arise from between the range and the horizon. Therefore for the third year the completeness is $\sim 20 \%$. Both Figures 2 and 3 show some regions of "overcompleteness" below $100 \mathrm{Mpc}$. This is probably due to local overdensities such as the Virgo Supercluster.

Our choice of $x_{\text {cut }}=x_{1 / 2}$ is motivated by a trade-off between including enough galaxies so that we encompass a reasonable fraction of putative sGRB host galaxies (e.g., Berger 2014; see his Figure 8) on the one hand, and not having incompleteness become too great an issue on the other hand. Taking $x_{\text {cut }}=x_{1 / 2}$ picks out about half of the sGRB host galaxies shown in Berger (2014), therefore the true efficiency factors for the three target years would be $\sim 50 \%, \sim 40 \%$, and $\sim 10 \%$, respectively, after convolving the $x>x_{1 / 2}$ completeness with the fact that $x_{1 / 2}$ lies near the median in the observed sGRB $x$ distribution.

Another caveat arises from the fact that by utilizing $B$-band data we are implicitly taking $B$-band luminosity as a proxy for the compact object merger rate. Although this assumption is generally supported by sGRB observations (Berger 2014), it may in fact not be a universally good proxy (e.g., de Freitas Pacheco et al. 2006; O'Shaughnessy et al. 2010; Hanna et al. 2014).

\section{GALAXY STRATEGY}

As regards to identifying a candidate host galaxy for a GW event, a galaxy catalog is only half of the picture. Of course the starting point is the $\mathrm{GW}$ localization. Initial localizations in the $\sim 2015$ time frame are expected to be $\sim 500 \mathrm{deg}^{2}$, decreasing to $\sim 20 \mathrm{deg}^{2}$ by $\sim 2020$ (Aasi et al. 2013; Singer et al. 2014; Berry et al. 2015). In terms of the depth, the NS-NS inspiral range should increase from $\sim 60 \mathrm{Mpc}$ to $\sim 180 \mathrm{Mpc}$ over the same time frame. A projected timeline of NS-NS inspiral range versus date is given by Aasi et al. (2013). One may obtain a good estimate using simple considerations. In the CLU catalog there are $N^{*}=27559$ galaxies brighter than $M_{B}{ }^{*}$, and $N_{1 / 2}=47438$ brighter than $M_{B}{ }_{1 / 2}$. If one restricts $N_{1 / 2}$ based on the limited sky areas and volumes relevant for the three target years, i.e., $500 \mathrm{deg}^{2} \times 60 \mathrm{Mpc}$ for $2015,100 \mathrm{deg}^{2} \times 120 \mathrm{Mpc}$ for 2017 , and $20 \mathrm{deg}^{2} \times 180 \mathrm{Mpc}$ for 2020 , one obtains $N_{\text {gal }}=26.0,36.4$, and 18.8, respectively. As noted previously, since these distances represent putative NS-NS inspiral ranges, for a localization at $\mu$ we must roughly double the totals to take into account going from range to horizon if the distance error is large (i.e., plus or minus a factor of two).

Realistic idealizations for aLIGO localizations have been undertaken by several recent groups that attempt to quantify the "volume reductions" which might be realized. Nissanke et al. (2010) use a Markov Chain Monte Carlo (MCMC) technique to calculate distance measurement errors associated with aLIGO localizations of astrophysical populations of NS-NS and $\mathrm{BH}-$ NS binaries, considering both isotropically oriented as well as beamed events. They take as their starting point a precise sky localization based on a coincident EM detection of the same GW event. They present Fisher-matrix-derived linear scalings for $\left[\Delta D_{L} /\right.$ True $D_{L}$ ] for the two populations, assuming four $\mathrm{GW}$ detectors. If the EM emission from the NS-NS merger providing the coincident EM signal is isotropic, they find that, in combination with the precise sky position, the distance to NS-NS binaries can be measured with a fractional error of $\sim 20 \%-60 \%$, with most events clustered near $\sim 20 \%-30 \%$. If the EM emission from the NS-NS merger is beamed, with a $\sim 25^{\circ}$ opening angle, then the error on the distance is reduced by a factor of $\sim 2$ and much of the high error tail is eliminated. $\mathrm{BH}-\mathrm{NS}$ events are measured more accurately: the distribution of fractional distance errors lies in the range $\sim 15 \%-50 \%$, with most events clustered near $\sim 15 \%-25 \%$. Nissanke et al. (2013) carry out extensive end-to-end simulations, looking at GW sky localization, distance errors, and volume errors using NS-NS and BH-NS mergers. They compare MCMC-derived distance measures with $3 \mathrm{D}$ volume measures. They outline optimal 
strategies to prepare for identifying EM counterparts of a GW merger.

L. P. Singer et al. (2016, in preparation) have created full 3D position reconstructions for a large population of simulated early aLIGO NS-NS events. They provide a simple approximation for the $3 \mathrm{D}$ distance distribution and qualitatively describe the shapes that emerge. In the present work, as we are more interested in the impact of the galaxy catalog we use an even simpler description: we assume that the localization subtends a given solid angle, and is a shell between two constant radii.

L. P. Singer et al. (2016, in preparation) calculate full 3D aLIGO reconstructions based on BAYESTAR, a rapid reconstruction method for BNS mergers, and LALInference, a full Bayesian parameter estimation code (Singer et al. 2014; Singer 2015; Veitch et al. 2015), for the near-future of aLIGO -2015 and 2016. In this work we consider a longer time frame, i.e., up to the full achievement of aLIGO design sensitivity. In order to calculate galaxy sky counts in error boxes of given areas on the sky, we take a simplified approach compared to Nissanke et al. (2010, 2013), and Singer (2015). Namely, rather than using a realistic 3D reconstruction, for a given line of sight (LOS) we take a simple top-hat windowing function. For our three putative aLIGO target years-2015, 2017, and 2020 - we adopt $\mu=60,120$, and $180 \mathrm{Mpc}$, respectively, as fiducial LVC NS-NS inspiral ranges.

We consider 1000 randomly selected LOSs over the sky, and then search the CLU catalog to find galaxies within an angular separation that would place them inside an error box on the sky of (i) $500 \mathrm{deg}^{2}$, (ii) $100 \mathrm{deg}^{2}$, or (iii) $20 \mathrm{deg}^{2}$ for the three cases. Although the actual sky-projected aLIGO localizations will be complicated, the more important factor is simply the total sky area involved. Only galaxies are considered for which their luminosity places them above the 50th percentile mark $x_{1 / 2}$. The CLU galaxy count in a radial bin is incremented only if $|(r-\mu) / \mu|<0.3$ (Hanna et al. 2014), i.e., if the galaxy lies within a thick spherical shell with $\Delta \mu / \mu=0.6$, where the NSNS inspiral range $\mu=60,120$, and $180 \mathrm{Mpc}$, respectively. This has the effect of essentially doubling the galaxy counts derived by truncating the integration at the range $\mu$, thereby mimicking the effect of the range-to-horizon mapping (Hanna et al. 2014).

Figure 4 shows the results of this experiment. Each point is the mean of the 1000 individual LOS values, and the error bar is the standard deviation. Two main points are worth noting. The most obvious is simply that larger error boxes yield more galaxies. The second is that galaxy counts are only added out to $1.3 \mu$, by construction. In reality, one would continue to pick up sources out to the horizon at $2.26 \mu$. The total number of sources for $d<2.26 \mu$ is twice that for $d<\mu$ despite the factor 11.5 increase in volume (as many binaries are not at favorable inclination). To fully model this would require a variable efficiency factor with distance between the range and horizon, hence the motivation for our simple top-hat method. The CLU totals out to $r=\mu$ (for a localization in each year at $\mu$ ) are $N_{\text {gal }}=17.7 \pm 5,22.5 \pm 4$, and $10.5 \pm 2$, respectively. As noted previously, the CLU is $\sim 100 \%$ complete above $x_{1 / 2}$ at $60 \mathrm{Mpc}, \sim 80 \%$ complete at $120 \mathrm{Mpc}$, and $\sim 40 \%$ complete at $180 \mathrm{Mpc}$. In addition, the NS-NS range-to-horizon mapping roughly doubles the totals for localizations $r \simeq \mu$ with a large radial uncertainty.

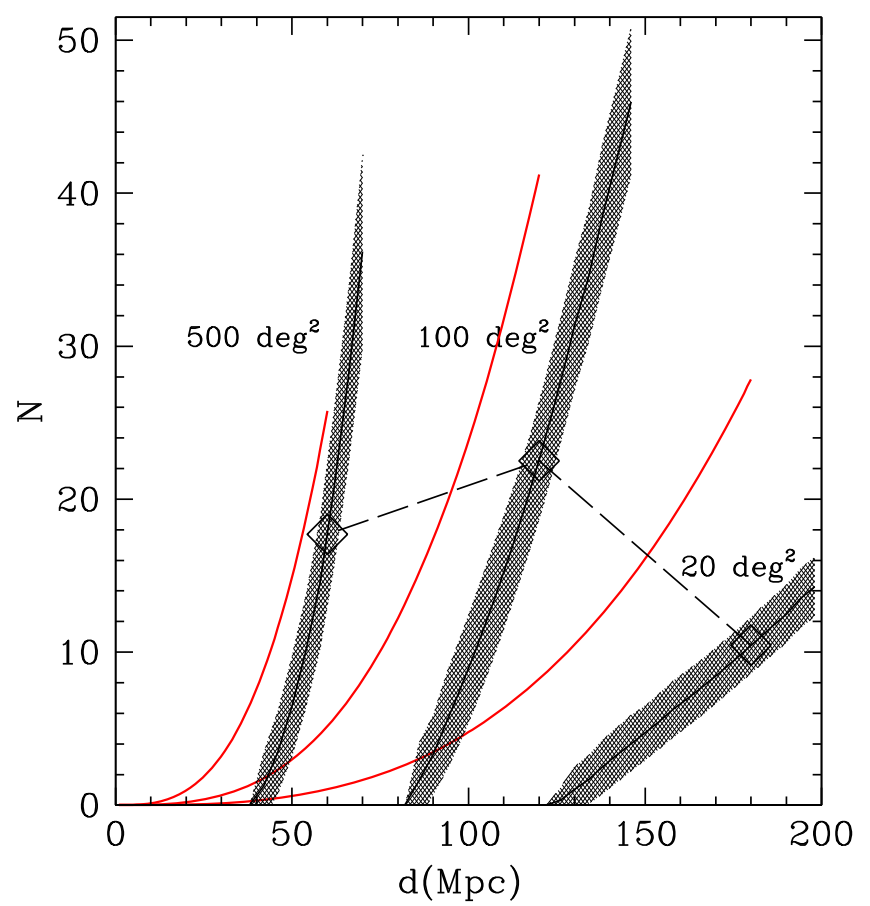

Figure 4. The number of CLU galaxies in a given size error box vs. range (black). In this experiment we implicitly assume a localization at the inspiral range for each of the three target years. Diamonds indicate the adopted NS-NS inspiral ranges for the three target years of this study, $\mu=60,120$, and $180 \mathrm{Mpc}$, respectively. The three curves are representative of the increasing localization capability of aLIGO+VIRGO with time, $\Delta \Omega \simeq 500 \mathrm{deg}^{2}$ (for 2015), $100 \mathrm{deg}^{2}$ (for 2017), and $20 \mathrm{deg}^{2}$ (for 2020). The CLU incompleteness weighting factors at $r=\mu$ are $1.0,1 / 0.8=1.25$, and $1 / 0.4$, respectively. The galaxy count totals indicated by the diamonds are $N_{\text {gal }}=17.7 \pm 5,22.5 \pm 4$, and $10.5 \pm 2$. We also present the corresponding $N_{\text {gal }}$ values determined directly from the Schechter function (red) weighted by the relevant volume $\Delta V=(4 / 3) \pi \mu^{3}(\Delta \Omega / 4 \pi)$ (see the end of Section 4).

For consistency we may consider galaxy counts derived directly from the Schechter function. The galaxy density above $x_{1 / 2}$ is $\int_{x_{1 / 2}}^{\infty} \phi^{*} x^{a} e^{-x} d x=2.35 \times 10^{-3} \mathrm{Mpc}^{-3}$. If we then multiply this by a volume $\Delta V=(4 / 3) \pi \mu^{3}(\Delta \Omega / 4 \pi)$, where, for the three target years $(\mu, \Delta \Omega)=\left(60 \mathrm{Mpc}, 500 \mathrm{deg}^{2}\right)$, $\left(120 \mathrm{Mpc}, 100 \mathrm{deg}^{2}\right)$, and $\left(180 \mathrm{Mpc}, 20 \mathrm{deg}^{2}\right)$, respectively, we obtain $N_{\text {gal }}=25.8,41.3$, and 27.8 .

\section{TILING OBSERVATIONS}

The results given in Figure 4 reveal the dramatic reduction in tiling requirements brought about by restricting a search methodology to the brighter galaxies. Were one simply to tile the entire 2015,2017 , and 2020 putative error boxes $A=500$, 100 , and $20 \mathrm{deg}^{2}$ using an EM detector with a field of view (FOV) of $\delta A=0.1 \mathrm{deg}^{2}$, i.e., $\sim 19 \times 19 \mathrm{arcmin}$, the number of tilings required would be $A / \delta A=5 \times 10^{3}, 10^{3}$, and $2 \times 10^{2}$, respectively.

Concentrating on selected bright galaxies reduces the tiling effort considerably. The optimal size of a "tile" is dictated by observations of short GRBs - in particular their locations within their host galaxies. Fong et al. (2010) use precise HST localizations to study the cumulative distribution of projected physical offsets for short GRBs with sub-arcsecond positions and find them to lie within $\sim 100 \mathrm{kpc}$ of their host galaxy centers. For a typical distance of interest in this study, $100 \mathrm{Mpc}$, this corresponds to $\sim 10^{-3}$ radian or $\sim 0.057 \mathrm{deg}-\mathrm{a}$ 
projected area on the sky of $\sim 0.010 \mathrm{deg}^{2}$ or $\sim 37 \mathrm{arcmin}^{2}$. This can be covered by an EM detector with a rectangular FOV of at least $7 \times 7$ arcmin or $49 \operatorname{arcmin}^{2}$. For such detectors the number of tilings will reduce to the number of galaxies as shown in Figure 4, i.e., $\sim 20, \sim 20$, and $\sim 10$, respectively. This represents a reduction in requisite tilings by $\sim 1-2$ orders of magnitude over a brute force methodology, i.e., simply covering the entire GW error box.

Furthermore, the underlying strategy of focusing attention only on bright galaxies is strengthened by the observation that short GRBs tend to lie in the larger, brighter galaxies (in contrast to long GRBs, which lie preferentially in dwarf irregulars). Fong et al. (2010) compare the projected physical offset distribution between short and long GRBs and find that, when normalized to the sizes of their host galaxies, the distributions are indistinguishable. However, the absolute length scales differ by a factor of $\sim 5$ (see also Fong \& Berger 2013). Berger (2014, see his Figure 8) plots all known sGRB host galaxy $x=L_{B} / L_{B}{ }^{*}$ values; they span a range $0.1 \lesssim x \lesssim 2$. Our adopted cut value in this study $x_{1 / 2} \simeq 0.6$ is roughly at the median of the observed distribution, therefore our bright galaxy strategy would pick up about half the sGRBs shown in Berger's sample. Therefore the total effective completeness would be reduced by another factor $\sim 2$ below that given in the previous section, which only considered completeness for $x>x_{1 / 2}$. The trade-off against lowering our cut value so as to include a greater fraction of putative sGRB host galaxies, i.e., using, for example, $x_{1 / 3}$ or $x_{1 / 4}$, is two-fold: (i) the number of galaxies would increase rapidly and (ii) incompleteness would become a more severe issue. A further caveat involves the issue of large SN kicks imparted to NS-NS binaries potentially leading to hostless events (Kelley et al. 2010; Behroozi et al. 2014).

\subsection{Optical}

Within an area of several square degrees on the sky there are many more optical transients than in other wavelengths, and therefore more opportunities for false positives (Kulkarni \& Kasliwal 2009; Nissanke et al. 2013; Drout et al. 2014; Tanaka et al. 2014; Cowperthwaite \& Berger 2015; L. P. Singer et al. 2016, in preparation). Most of this activity stems from variable sources within our own galaxy. If we restrict our attention to bright galaxies, false positives could still arise from powerful sources within the target galaxy such as SNe. However, SNe are rare and also have longer timescales than EM counterparts to NS binary mergers. More generally, Nissanke et al. (2013) and Kasliwal \& Nissanke (2014) show that false positives can be due to both foreground stars (e.g., flare stars and cataclysmic variables) and background galaxies (e.g., SNe and AGNs). Chance associations of such transients with a host galaxy location would be problematic to exclude based solely on photometry, and would probably necessitate multi-wavelength observations. An advantage with the optical (and near-IR) is that the emission of interest is due to the kilonova, which is quasi-isotropic, whereas X-ray emission, for instance, would be beamed.

A variety of large optical telescopes that have been active in GRB follow-up have large FOVs which would make them amenable to $\mathrm{GW}+\mathrm{EM}$ tiling observations: GTC/OSIRIS (10.4 $\mathrm{m}$ aperture-7.8 $\times 8.5 \mathrm{arcmin}$ FOV), Keck/LRIS $(10 \mathrm{~m}$ $-6 \times 8$ arcmin $), \quad$ LBT/LBC $(8.4 \mathrm{~m}-23 \times 23$ arcmin $)$, and
VLT/VIMOS $(8.2 \mathrm{~m}-14 \times 14 \mathrm{arcmin})$. These instruments could all cover the Fong et al. (2010) short GRB projected area in one tile.

\section{2. $X-R a y$}

The transient sky is less chaotic in X-rays than in the optical. Therefore individual transients stand out more. However, for short GRBs only the beamed events will be detected in X-rays, which reduces the detection chances by the reciprocal of the beaming factor $f_{b}$ of a short GRB, which is highly uncertain, $f_{b}^{-1} \simeq 10-100$. The primary instrument of relevance for X-ray follow-up is the Swift/XRT (Burrows et al. 2005), a focusing XRT with a $110 \mathrm{~cm}^{2}$ effective area, an 18 arcsec resolution (one-half power diameter) in the $0.2-10 \mathrm{keV}$ band, and a fieldof-view (FOV) of $23.6 \times 23.6 \mathrm{arcmin}$, or $\sim 0.15 \mathrm{deg}^{2}$. Thus one XRT tile would be $\delta A \sim 0.15 \mathrm{deg}^{2}$, or about 10 times the minimum required to tile a putative short GRB host galaxy. Given an XRT exposure time of $\sim 0.1 \mathrm{ks}$ (Kanner et al. 2012) and a comparable time to slew between tiles, a complete search of the three error boxes depicted in Figure 4 would place modest demands on Swift. The bright galaxy strategy for GWEM follow-up described in this work results in a far less strenuous use of XRT resources than discussed, for example, in Evans et al. (2016).

\subsection{Radio}

Explosive transients that eject ionized matter into a surrounding medium eventually produce radio waves via synchrotron radiation as the electrons interact with tangled magnetic fields in shocked regions. Radio emission has been seen in GRBs, SN remnants, colliding winds in massive binaries, symbiotic stars, and cataclysmic variables. Over long timescales the radio provides good calorimetry on the total energetics of the explosion due to its quasi-isotropic emission (Nakar \& Piran 2011; Hotokezaka \& Piran 2015). Thus, as with the optical observations of kilonovae, the radio band also has an advantage over X-rays, which are beamed. The radio sky is also relatively quiet for fast transients, although this may be at least partly due to the lower sensitivity of transient radio surveys compared to optical or X-ray ones. (Frail et al. 2012; Mooley et al. 2013; Mooley et al. 2016).

Radio facilities have done extensive follow-up work on GRBs and would be relevant in radio tilings of GW error boxes insofar as having beam FWHMs that would cover the expected region in an $L \simeq L^{*}$ galaxy: e.g., AMI-LA: 6 arcmin beam at $16 \mathrm{GHz}$ and JVLA: 9 arcmin beam in $\mathrm{C}$ band $(4.5-5 \mathrm{GHz})$. At least 4 short GRBs have had radio afterglows detected, 051221a, 050724, 130603b, and 140903 (Chandra \& Frail 2012; Berger 2014). They were all quite faint, $\sim 100 \mu \mathrm{Jy}$, but they were also considerably beyond the range of current interest, $d \leqslant 200 \mathrm{Mpc}$ or $z \leqslant 0.047$.

\section{DISCUSSION AND CONCLUSION}

In this work we have argued for a strategy in which only the brighter galaxies are considered in GW-EM follow-ups. We show that the CLU catalog is fairly complete for $x>x_{1 / 2}$ out to $200 \mathrm{Mpc}$. By weighting the galaxy counts within projected sky areas with a simple model for the aLIGO radial localization, we find that only about $18 \pm 5$ (for 2015), $23 \pm 4$ (for 2017), or $11 \pm 2$ (for 2020) galaxies need to be 
considered, assuming error boxes of 500, 100 and $20 \mathrm{deg}^{2}$, respectively. This results, if one restricts attention to galaxies in the upper 50th percentile, in integrated luminosity density. Furthermore, there are numerous EM detectors with the property that one tile (i.e., one FOV) would encompass the region of interest. These facilities - optical, X-ray, and radio detectors-have carried out GRB follow-up previously and could participate in future tiling observations. Having one tile per galaxy reduces the number of requisite tiles down to the number of galaxies, a reduction in tiling effort by $\sim 1-2$ orders of magnitude.

The CLU efficiencies for $\mathrm{x}>x_{1 / 2}$ vary from $\sim 100 \%$ at the NS-NS inspiral range $\mu=60 \mathrm{Mpc}$ to $\sim 40 \%$ at $\mu=180 \mathrm{Mpc}$. For our third target year, 2020, the efficiency is cut by an additional factor of two due to the fact that $\sim$ half of the detections at any given time come from beyond the range, and the CLU is truncated beyond $200 \mathrm{Mpc}$. In addition to the CLU incompleteness, our adopted cut $x_{\mathrm{cut}}=x_{1 / 2}$ lies at roughly the median in the observed sGRB host galaxy $L_{B} / L_{B}{ }^{*}$ distribution (Berger 2014), therefore we lose another factor of two in potential sGRB hosts. Lowering our $x_{\text {cut }}$ value further would allow us to cover more of the expected sGRB host galaxy $L_{B} / L_{B}{ }^{*}$ distribution but would exacerbate the CLU incompleteness issue. An additional caveat arises from the hostless sGRBs with optical afterglows, and the (majority) of sGRBs for which no afterglows have been observed. If these lie further from the host galaxy centers than the $\sim 100 \mathrm{kpc}$ maximal offset indicated by Fong and Berger et al. (2013), then our strategy could miss the kilonova emission.

M.M.K. acknowledges generous support from the CarnegiePrinceton Fellowship. S.N. and L.P.S. thank the Aspen Center for Physics and the NSF Grant \#1066293 for hospitality during the editing of this paper. S.N. acknowledges generous support from the Radboud University Excellence Initiative. We thank internal LIGO reviewer Ilya Mandel for excellent feedback on all aspects of the paper.

\section{REFERENCES}

Aasi, J., Abadie, J., Abbott, B. P., et al. (The LIGO Scientific Collaboration) 2013, arXiv:astro-ph/1304.0670v1

Aasi, J., Abbott, B. P., Abbott, R., et al. (The LIGO Scientific Collaboration) 2015, CQGra, 32, 074001

Abadie, J., Abbott, B. P., Abbott, R., et al. (The LIGO Scientific Collaboration) 2010, CQGra, 27, 173001

Acernese, F., Agathos, M., Agatsuma, K., et al. (Virgo Collaboration) 2015, CQGra, 32, 024001

Ade, P. A. R., Aghanim, N., Armitage-Caplan, C., et al. 2014, A\&A, 571, A16 Allen, C. W. 1973, Astrophysical Quantities (Athlone: Univ. London)

Barnes, J., \& Kasen, D. 2013, ApJ, 775, 18

Bauswein, A., Goriely, S., \& Janka, H.-T. 2013, ApJ, 773, 78

Behroozi, P. S., Ramirez-Ruiz, E., \& Fryer, C. L. 2014, ApJ, 792, 123

Belczynski, K., Kalogera, V., \& Bulik, T. 2002, ApJ, 572, 407

Berger, E. 2014, ARA\&A, 52, 43

Berger, E., Fong, W., \& Chornock, R. 2013, ApJL, 774, L23

Berry, C. P. L., Mandel, I., Middleton, H., et al. 2015, ApJ, 804, 114

Bonne, N. J., Brown, M. J. I., Jones, H., \& Pimbblet, K. A. 2015, ApJ, 799, 160

Burrows, D. N., Grupe, D., Capalbi, M., et al. 2006, ApJ, 653, 468

Burrows, D. N., Hill, J. E., Nousek, J. A., et al. 2005, SSRv, 120, 165

Carroll, B. W., \& Ostlie, D. A. 1996, An Introduction to Modern Astrophysics (New York: Addison-Wesley)

Chan, M. L., Hu, Y.-M., Messenger, C., Hendry, M., \& Heng, I. S. 2015, arXiv:astro-ph/1506.04035

Chandra, P., \& Frail, D. A. 2012, ApJ, 746, 156

Chen, H.-Y., \& Holz, D. E. 2013, PhRvL, 111, 181101
Chen, H.-Y., \& Holz, D. E. 2015, arXiv:astro-ph/1509.00055

Coward, D. M., Howell, E. J., Piran, T., et al. 2012, MNRAS, 425, 2668

Cowperthwaite, P. S., \& Berger, E. 2015, ApJ, 814, 25

Dalal, N., Holz, D. E., Hughes, S. A., \& Jain, B. 2006, PhRvD, 74, 063006

de Freitas Pacheco, J. A., Regimbau, T., Vincent, S., \& Spallicci, A. 2006, IJMPD, 15, 235

Dominik, M., Belczynski, K., Fryer, C., et al. 2013, ApJ, 779, 72

Driver, S. P., Liske, J., Cross, N. J. G., De Propris, R., \& Allen, P. D. 2005 , MNRAS, 360, 81

Drout, M. R., Chornock, R., Soderberg, A. M., et al. 2014, ApJ, 794, 23

East, W. E., \& Pretorius, F. 2012, ApJL, 760, L4

Eichler, D., Livio, M., Piran, T., \& Schramm, D. N. 1989, Natur, 340, 126

Evans, P. A., Osborne, J. P., Kennea, J. A., et al. 2016, MNRAS, 455, 1522

Fan, X., Messenger, C., \& Heng, I. S. 2015, ApSSP, 40, 35

Fernández, R., Kasen, D., Metzger, B. D., \& Quataert, E. 2015, MNRAS, 446, 750

Fernández, R., \& Metzger, B. D. 2013, MNRAS, 435, 502

Finn, L. S. 1996, PhRvD, 53, 2878

Finn, L. S., \& Chernoff, D. F. 1993, PhRvD, 47, 2198

Fong, W., \& Berger, E. 2013, ApJ, 776, 18

Fong, W., Berger, E., \& Fox, D. B. 2010, ApJ, 708, 9

Fong, W., Berger, E., Margutti, R., et al. 2012, ApJ, 756, 189

Foucart, F., Duez, M. D., Kidder, L. E., \& Teukolsky, S. A. 2011, PhRvD, 83, 024005

Foucart, F., O'Connor, E., Roberts, L., et al. 2015, PhRvD, 91, 124021

Frail, D. A., Kulkarni, S. R., Ofek, E. O., Bower, G. C., \& Nakar, E. 2012, ApJ, 747, 70

Gehrels, N., Chincarini, G., Giommi, P., et al. 2004, ApJ, 611, 1005

Ghosh, S., \& Nelemans, G. 2015, ApSSP, 40, 51

González, R. E., Lares, M., Lambas, D. G., \& Valotto, C. 2006, A\&A, 445, 51

Grossman, D., Korobkin, O., Rosswog, S., \& Piran, T. 2014, MNRAS, 439, 757

Grupe, D., Burrows, D. N., Patel, S. K., et al. 2006, ApJ, 653, 462

Hanna, C., Mandel, I., \& Vousden, W. 2014, ApJ, 784, 8

Hotokezaka, K., Kiuchi, K., Kyutoku, K., et al. 2013, PhRvD, 87, 024001

Hotokezaka, K., \& Piran, T. 2015, MNRAS, 450, 1430

Huchra, J. P., Macri, L. M., Masters, K. L., et al. 2012, ApJS, 199, 26

Kanner, J., Camp, J., Racusin, J., Gehrels, N., \& White, D. 2012, ApJ, 759, 22

Kasen, D., Badnell, N. R., \& Barnes, J. 2013, ApJ, 774, 25

Kasen, D., Fernández, R., \& Metzger, B. D. 2015, MNRAS, 450, 1777

Kasliwal, M. M., \& Nissanke, S. 2014, ApJL, 789, L5

Kelley, L. Z., Mandel, I., \& Ramirez-Ruiz, E. 2013, PhRvD, 87, 123004

Kelley, L. Z., Ramirez-Ruiz, E., Zemp, M., Diemand, J., \& Mandel, I. 2010, ApJL, 725, L91

Kouveliotou, C., Meegan, C. A., Fishman, G. J., et al. 1993, ApJL, 413, L101

Kulkarni, S., \& Kasliwal, M. M. 2009, in Proc. RIKEN Symp., Astrophysics with All-Sky X-Ray Observations, ed. N. Kawai et al. (Saitama: RIKEN), 312

Kyutoku, K., Ioka, K., \& Shibata, M. 2013, PhRvD, 88, 041503

Li, L.-X., \& Paczyński, B. 1998, ApJL, 507, L59

Liske, J., Lemon, D. J., Driver, S. P., Cross, N. J. G., \& Couch, W. J. 2003 , MNRAS, 344, 307

Maggiore, M. 2007, Gravitational Waves, Vol. 1 (Oxford: Oxford Univ. Press)

Metzger, B. D., Bauswein, A., Goriely, S., \& Kasen, D. 2015, MNRAS, 446, 1115

Metzger, B. D., \& Berger, E. 2012, ApJ, 746, 48

Metzger, B. D., Martínez-Pinedo, G., Darbha, S., et al. 2010, MNRAS, 406, 2650

Mooley, K. P., Hallinan, G., Bourke, S., et al. 2016, ApJ, 818, 105

Mooley, K. P., Frail, D. A., Ofek, E. O., et al. 2013, ApJ, 768, 165

Nakar, E., \& Piran, T. 2011, Natur, 478, 82

Narayan, R., Paczyński, B., \& Piran, T. 1992, ApJL, 395, L83

Nissanke, S., Holz, D. E., Hughes, S. A., Dalal, N., \& Sievers, J. L. 2010, ApJ, 725,496

Nissanke, S., Kasliwal, M., \& Georgieva, A. 2013, ApJ, 767, 124

Norberg, P., Cole, S., Baugh, C. M., et al. 2002, MNRAS, 336, 907

Nuttall, L. K., \& Sutton, P. J. 2010, PhRvD, 82, 102002

O'Shaughnessy, R., Kalogera, V., \& Belczynski, K. 2010, ApJ, 716, 615

O’Shaughnessy, R., \& Kim, C. 2010, ApJ, 715, 230

Ott, C. D., Abdikamalov, E., Mösta, P., et al. 2013, ApJ, 768, 115

Phinney, E. S. 1991, ApJL, 380, L17

Piran, T., Nakar, E., \& Rosswog, S. 2013, MNRAS, 430, 2121

Roberts, L. F., Kasen, D., Lee, W. H., \& Ramirez-Ruiz, E. 2011, ApJL, 736, L21

Schechter, P. 1976, ApJ, 203, 297 
Schutz, B. F. 2011, CQGra, 28, 125023

Sekiguchi, Y., Kiuchi, K., Kyutoku, K., \& Shibata, M. 2015, PhRvD, 91, 064059

Siellez, K., Boër, M., \& Gendre, B. 2014, MNRAS, 437, 649

Singer, L. P. 2015, PhD thesis, California Institute of Technology

Singer, L. P., Price, L. R., Farr, B., et al. 2014, ApJ, 795, 105

Skrutskie, M. F., Cutri, R. M., Stiening, R., et al. 2006, AJ, 131, 1163
Tanaka, M., \& Hotokezaka, K. 2013, ApJ, 775, 113

Tanaka, M., Hotokezaka, K., Kyutoku, K., et al. 2014, ApJ, 780, 31

Tanvir, N. R., Levan, A. J., Fruchter, A. S., et al. 2013, Natur, 500, 547

Veitch, J., Raymond, V., Farr, B., et al. 2015, PhRvD, 91, 042003

Wanajo, S., Sekiguchi, Y., Nishimura, N., et al. 2014, ApJL, 789, L39

Wanderman, D., \& Piran, T. 2015, MNRAS, 448, 3026

White, D. J., Daw, E. J., \& Dhillon, V. S. 2011, CQGra, 28, 085016 